\title{
Experiências sexuais após prostatectomia radical não poupadora de nervos
}

Sexual experiences after non-nerve sparing radical prostatectomy Experiencias sexuales después de prostatectomía radical sin conservación de bandeletas neurovasculares

Álvaro Martínez-Bordajandi' io ittps://orcid.org/0000-0003-3398-5080

Cayetano Fernández-Sola² ${ }^{2}$ hittps://orcid.org/0000-0003-1721-0947

Ana Patricia Puga-Mendoza ${ }^{3}$ io https://orcid.org/0000-0002-8178-2088

Olga María López-Entrambasaguas ${ }^{4}$ is https://orcid.org/0000-0001-7592-3553

Manuel Lucas-Matheu ${ }^{5}$ io hitps://orcid.org/0000-0002-1551-5854

José Granero-Molina² io https://orcid.org/0000-0002-7051-2584

Isabel María Fernández Medina² io https://orid. org/0000-0003-0805-1542 José Manuel Hernández-Padillata ${ }^{2,6}$ io hitps://orcid. org/0000-0002-5032-9440

\section{Como citar:}

Martínez-Bordajandi A, Fernández-Sola C, Puga-Mendoza AP, López-Entrambasaguas OM, Lucas-Matheu M, Granero-Molina J, et al. Experiências sexuais após prostatectomia radical não poupadora de nervos . Acta Paul Enferm. 2020;33:AAPE20190237.

DOI

http://dx.doi.org/10.37689/acta-

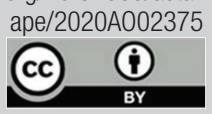

Descritores

Neoplasias da próstata; Prostatectomia; Saúde do homen; Comportamento sexual; Sexualidade

Keywords Prostatic neoplasms; Prostatectomy; Sexuality; Men's health; Sexual behavior; Sexuality

Descriptores

Neoplasias de la próstata; Prostatectomía; Salud del hombre; Conducta sexual; Sexualidad

\section{Submetido \\ 12 de Agosto de 2019 \\ Aceito \\ 3 de Fevereiro de 2020}

\section{Autor correspondente}

Cayetano Fernández-Sola Email: cfernan@ual.es

\section{Resumo}

Objetivo: 0 objetivo deste estudo foi explorar as experiências e expectativas de pacientes submetidos a prostatectomia radical não poupadora de nervos.

Métodos: Estudo qualitativo, descritivo-exploratório, baseado na fenomenologia hermenêutica. Foram realizadas dezesseis entrevistas em profundidade entre fevereiro e dezembro de 2017 e análise indutiva dos dados.

Resultados: Dois temas que refletem as experiências dos pacientes emergiram da análise: (1) As mudanças sexuais como fator-chave da resposta adaptativa, com os subtemas "Dificuldades sexuais após a cirurgia" e "Questões com relacionamentos íntimos e bem-estar psicossocial"; (2) Prostatectomia: a necessidade de adaptação a uma sexualidade holística, com os subtemas "Questionamento das experiências a partir do comportamento sexual atual" e "Adaptação da sexualidade e a intimidade após a cirurgia".

Conclusão: Pacientes submetidos à prostatectomia enfrentam dificuldades sexuais como disfunção erétil. Essas mudanças causam problemas nos relacionamentos sexuais e íntimos que afetam seu bem-estar. Diante dessa situação, alguns participantes questionam suas práticas sexuais habituais e tentam se adaptar a uma sexualidade holística não centrada no coito, incorporando formas inovadoras de sexualidade.

Conhecer as experiências de homens submetidos a prostatectomia radical pode ajudar os profissionais de saúde a oferecer novas estratégias para lidar com as mudanças ambientais envolvidas na cirurgia da próstata.

\section{Abstract}

Objective: The aim of this study was to explore the experiences and expectations of patients who underwent non-nerve sparing radical prostatectomy.

Methods: A qualitative descriptive, exploratory, study, based on hermeneutic phenomenology. Sixteen in-depth interviews were carried out between February and December 2017. An inductive analysis of data was performed. Results: Two themes reflecting the patients' experiences emerged from the analysis: (1) Sexual changes as a key factor of the adaptive response, with the subthemes "Sexual difficulties after surgery" and "Issues with intimate relationships and psychosocial wellbeing". (2) Prostatectomy: the need of adaptation to a holistic sexuality, with the subthemes "Questioning the experiences from the current sexual behavior" and "Adapting sexuality and intimacy after surgery".

Conclusion: Patients undergoing prostatectomy face sexual difficulties such as erectile dysfunction. These changes cause problems in sexual and intimate relationships that affect their wellbeing. Given this situation,

Surgical Emergency Unit, Oxford University Hospitals NHS Foundation Trust, Oxford, Reino Unido.

2Department of Nursing Science, Physiotherapy and Medicine, University of Almeria, Almeria, Espanha.

${ }^{3}$ Oxford University Hospitals NHS Foundation Trust, Oxford, Reino Unido.

${ }^{4}$ Departamento de Enfermería, Universidad de Jaen, Jaen, Espanha.

${ }^{5}$ Facultad de Ciencias de la Salud, University of Almeria, Almeria, Espanhan

${ }^{6}$ Adult, Child and Midwifery Department, School of Health and Education, Middlesex University, London, Reino Unido.

Conflitos de interesse: nada a declarar. 
some participants question their usual sexual practices and try to adapt to a holistic sexuality not centered on coitus by incorporating innovative forms of sexuality.

Knowing the experiences of men who underwent radical prostatectomy might help healthcare workers to provide new strategies for coping with the environmental changes involved in prostate surgery.

\section{Resumen}

Objetivo: El objetivo de este estudio fue analizar las experiencias y expectativas de pacientes sometidos a prostatectomía radical sin conservación de bandeletas neurovasculares.

Métodos: Estudio cualitativo, descriptivo-exploratorio, basado en la fenomenología hermenéutica. Se realizaron 16 entrevistas en profundidad entre febrero y diciembre de 2017 y un análisis inductivo de los datos.

Resultados: A partir del análisis, surgieron dos temas que reflejan las experiencias de los pacientes: (1) Los cambios sexuales como factor clave de la respuesta adaptativa, con dos subtemas "Dificultades sexuales después de la cirugía" y "Cuestiones sobre relaciones íntimas y bienestar psicosocial" y (2) Prostatectomía: necesidad de adaptación a una sexualidad holística, con los subtemas "Cuestionamiento de las experiencias a partir del comportamiento sexual actual" y "Adaptación de la sexualidad e intimidad después de la cirugía".

Conclusión: Los pacientes sometidos a prostatectomía enfrentan dificultades sexuales como disfunción eréctil. Estos cambios causan problemas en las relaciones sexuales e íntimas que afectan su bienestar. Ante esta situación, algunos participantes cuestionan sus prácticas sexuales habituales e intentan adaptarse a una sexualidad holística no centrada en el coito, incorporando formas innovadoras de sexualidad.

Conocer las experiencias de hombres sometidos a prostatectomía radical puede ayudar a los profesionales de la salud a ofrecer nuevas estrategias para lidiar con los cambios ambientales relacionados con la cirugía de próstata.

\section{Introdução}

O câncer de próstata é o segundo câncer mais prevalente no mundo ${ }^{(1)}$ e o tipo mais comum de câncer entre os homens na Europa.(2) $\mathrm{Na}$ Espanha, corresponde a $12,9 \%$ de todos os diagnósticos de câncer, o que representou cerca de 33.370 novos casos em 2015. ${ }^{(3)}$ A taxa de mortalidade é de 9,2\% na Espanha (taxa padronizada por idade). ${ }^{(2)}$ Quando a doença é localizada, o objetivo do tratamento é remover o tumor por radiação ${ }^{(4)}$ ou excisão cirúrgica. ${ }^{(5)}$ Os casos de metástase exigem tratamento sistêmico, como quimioterapia, imunoterapia ou terapia hormonal, para melhorar a sobrevida. ${ }^{(6)}$ A prostatectomia radical está entre as opçóes de tratamento cirúrgico ${ }^{(7,8)}$ e os pacientes submetidos a esse procedimento tiveram um aumento de 2,9 anos na expectativa de vida ${ }^{(7)}$ $\mathrm{Na}$ prostatectomia radical poupadora de nervos, os nervos periprostáticos são preservados, ${ }^{(9)}$ embora isso nem sempre seja possível. Como depende da extensão do tumor, algumas vezes é realizada uma prostatectomia radical não poupadora de nervos. ${ }^{(10)}$

A prostatectomia radical não poupadora de nervos tem sido associada com a disfunção erétil, ${ }^{(11)}$ problemas ejaculatórios e disfunçóes orgásmicas. ${ }^{(12)}$ Também foi descrita a perda de libido. ${ }^{(13)}$ Após uma prostatectomia radical não poupadora de nervos, os pacientes precisam estar atentos a aspectos físicos, psicológicos e informacionais. ${ }^{(14)}$ Esquecer as necessidades sexuais dos indivíduos pode afetar seu desenvolvimento e autoconceito. ${ }^{(15)}$ Por exemplo, a disfunção erétil ligada com a prostatectomia radical tem sido associada a diminuiçáo da masculinidade. ${ }^{(16)}$ Esta tem sido relatada como uma das experiências mais angustiantes no pós-operatório, ${ }^{(17)}$ portanto, os profissionais de saúde devem avaliar regularmente as necessidades sexuais desses indivíduos. ${ }^{(18)} \mathrm{O}$ cuidado integral aos pacientes prostatectomizados é um desafio para os profissionais, ${ }^{(19)}$ o que demonstra a importância do treinamento dos cuidadores de saúde no manejo de problemas sexuais. ${ }^{(20)}$ Nos últimos anos, surgiu a necessidade de estudos qualitativos sobre a dimensão sexual e psicossocial desse problema. ${ }^{(21)}$ As questóes de pesquisa que nortearam o presente estudo foram: quais são as experiências sexuais de pacientes submetidos a uma prostatectomia radical não poupadora de nervos? Quais são as expectativas dos pacientes submetidos a uma prostatectomia radical não poupadora de nervos em relação a sua sexualidade?

O objetivo deste estudo foi analisar as experiências e expectativas de pacientes submetidos a prostatectomia radical náo poupadora de nervos.

\section{Métodos}

\section{Desenho do estudo}

Estudo exploratório, qualitativo, descritivo, baseado na fenomenologia hermenêutica de Gadame. ${ }^{(22)}$ 
Esse desenho é usado em pesquisas sobre sexualidade humana. ${ }^{(23)}$ A compreensão da narrativa dos participantes requer preparo para um diálogo a partir do qual irão emergir diferentes significados. ${ }^{(22)}$

\section{Local e população}

Este estudo foi realizado na sede local da Associação Espanhola contra o Câncer - AECC (Asociación Española contra el Cáncer). Os participantes foram recrutados através de uma técnica de amostragem por conveniência. Os critérios de inclusão foram: 1) ter sido submetido a uma prostatectomia radical não poupadora de nervos como tratamento para câncer de próstata antes de 2016, de acordo com o relatório de alta hospitalar dos participantes; 2) assinar o termo de consentimento esclarecido para participação no estudo; 3) ter capacidade cognitiva; e 4) ter uma percepção adequada da função sexual antes do procedimento. Os critérios de exclusão foram: 1) ter algum comprometimento cognitivo que interferisse no entendimento e na resposta às perguntas; e 2) receber tratamento que interferisse na função sexual, como terapia hormonal. A amostra final foi composta por 16 participantes de um total de 24 sujeitos contatados. Oito pessoas foram excluídas da amostra por não atenderem aos critérios de inclusão/exclusão: dois homens mantiveram sua integridade sexual após a cirurgia, um estava em terapia hormonal no momento da coleta de dados e cinco homens relataram função sexual inadequada no pré-operatório. Depois que os participantes co- meçaram a repetir os mesmos conceitos e nenhum dado adicional foi revelado para o desenvolvimento de novas categorias, os pesquisadores consideraram que a saturação dos dados tinha sido alcançada e concluíram a coleta de dados.

\section{Definição de amostra}

A idade média dos participantes foi de 64,2 anos (desvio padrão=4,16). Em média, eles fizeram cirurgia 4,9 anos antes do estudo. Os dados sociodemográficos dos participantes estão na tabela 1 .

\section{Coleta e análise de dados}

$\mathrm{O}$ período de coleta de dados foi entre fevereiro e dezembro de 2017. Foram realizadas dezesseis entrevistas em profundidade. $\mathrm{O}$ pesquisador principal contatou os integrantes da amostra por telefone e explicou o objetivo do estudo. Os pacientes que atenderam aos critérios e aceitaram participar foram selecionados. Foi agendada uma reunião para a realização das entrevistas individuais pelo pesquisador principal, que tinha formação anterior em metodologia de pesquisa qualitativa (Mestrado em Pesquisa). Foi seguido um protocolo de entrevista contendo os objetivos, aspectos éticos e um guia/ roteiro de perguntas. $\mathrm{O}$ protocolo da entrevista foi ensaiado previamente e o entrevistador foi instruído a conduzir as entrevistas de forma que se assemelhassem a uma conversa aprofundada. Para construir confiança, inicialmente, os participantes foram questionados sobre o impacto da prostatectomia

Tabela 1. Dados sociodemográficos dos participantes

\begin{tabular}{|c|c|c|c|c|c|c|}
\hline Entrevista & Idade & Nível educacional & Estado civil & $\begin{array}{l}\text { Data da } \\
\text { prostatectomia }\end{array}$ & Orientação sexual & Tipo de cirurgia \\
\hline P-01 & 74 & Básico & Casado & Fevereiro, 2014 & Heterossexual & Prostatectomia radical perineal \\
\hline P-02 & 65 & Nenhum estudo & Casado & Março, 2006 & Heterossexual & Prostatectomia radical retropúbica \\
\hline P-03 & 60 & Médio & Casado & Setembro, 2008 & Heterossexual & Prostatectomia radical perineal \\
\hline P-04 & 62 & Nenhum estudo & Em um relacionamento & Fevereiro, 2010 & Heterossexual & Prostatectomia radical retropúbica \\
\hline P-05 & 68 & Médio & Casado & Agosto, 2012 & Heterossexual & Prostatectomia radical retropúbica \\
\hline P-06 & 60 & Básico & Casado & Janeiro, 2015 & Heterossexual & Prostatectomia radical retropúbica \\
\hline P-07 & 62 & Superior & Casado & Abril, 2015 & Heterossexual & Prostatectomia radical retropúbica \\
\hline P-08 & 64 & Superior & Em um relacionamento & Novembro, 2009 & Heterossexual & Prostatectomia radical retropúbica \\
\hline P-09 & 59 & Superior & Casado & Abril, 2014 & Heterossexual & Prostatectomia radical retropúbica \\
\hline P-10 & 65 & Médio & Em um relacionamento & Dezembro, 2011 & Heterossexual & Prostatectomia radical perineal \\
\hline P-11 & 69 & Básico & Casado & Janeiro, 2008 & Heterossexual & Prostatectomia radical perineal \\
\hline P-12 & 63 & Superior & Em um relacionamento & Setembro, 2010 & Heterossexual & Prostatectomia radical retropúbica \\
\hline P-13 & 60 & Básico & Casado & Junho, 2011 & Heterossexual & Prostatectomia radical retropúbica \\
\hline P-14 & 71 & Médio & Casado & Janeiro, 2012 & Heterossexual & Prostatectomia radical perineal \\
\hline P-15 & 66 & Básico & Em um relacionamento & Fevereiro, 2009 & Heterossexual & Prostatectomia radical retropúbica \\
\hline P-16 & 63 & Superior & Casado & Novembro, 2013 & Heterossexual & Prostatectomia radical perineal \\
\hline
\end{tabular}


radical em sua qualidade de vida. Quando o pesquisador sentiu a atmosfera de confiança, foram introduzidas questóes relacionadas a atividade sexual, por exemplo, "Você poderia me dizer como suas experiências sexuais mudaram após a operação?".

As entrevistas tiveram duração média de 48 minutos, foram gravadas digitalmente e transcritas manualmente. Na sequência, foi feita a revisão dos dados que, juntamente com as anotaçóes de campo e os comentários do entrevistador, constituíram a unidade hermenêutica de análise.

A análise das entrevistas foi baseada nas etapas a saber: $^{(23)}$ (1) Decidir se a questão do estudo era pertinente em relação aos pressupostos metodológicos. (2) Identificar a pré-compreensão dos pesquisadores sobre o objeto em estudo. No presente estudo, a pré-compreensão dos pesquisadores resultou da experiência clínica do pesquisador principal com pacientes prostatectomizados em unidades de emergência em cirurgia e urologia. (3) Adquirir compreensão, através do diálogo com os pacientes: durante as conversas, foi alcançado um entendimento espontâneo sobre o que os pacientes estavam expressando, que foi ampliado com mais perguntas para esclarecimento. Por exemplo, "O que exatamente você quer dizer quando menciona o medo de não se sentir mais homem?" (4) Compreender o fenômeno através do diálogo com o texto. As entrevistas foram transcritas imediatamente pelo pesquisador para evitar perder ou esquecer detalhes. As transcrições foram lidas na íntegra para extração da ideia geral, em seguida, relidas linha por linha, o significado de cada sentença foi estudado, e pesquisados os temas, subtemas e unidades de significado. Três pesquisadores triangularam a análise e foram excluídas quaisquer interpretaçôes (códigos, subtemas e temas) em que não houvesse acordo. Somente as citaçóes mais representativas para cada subtema foram selecionadas (por consenso) para inclusão no relatório. Nesta etapa, foi utilizado o software ATLAS.ti 8. Para este fim, foi desenvolvido um projeto ao qual foram adicionadas todas as transcriçôes. O processo de codificação foi realizado com as ferramentas do software (codificação aberta e codificação por lista) e construção de redes para facilitar o estabelecimento de relaçóes entre códigos, temas e subtemas (Figura 1).
Rigor: Para garantir o rigor do estudo, foi obtida validade interna através da representação de todas as opinióes dos participantes. A lista final de temas, subtemas e unidades de significado foi entregue aos participantes para aprovação e confirmação de que se sentiam identificados com a interpretaçáo. A confiabilidade foi reforçada pela triangulação de dados realizada pelos pesquisadores no processo de análise. O checklist Consolidated Criteria for Reporting Qualitative Research (COREQ), amplamente aceito na literatura científica, foi utilizado para garantir a validade científica. ${ }^{(24)}$

\section{Aspectos éticos}

Os participantes foram informados sobre o objetivo do estudo, de que sua participação era voluntária e poderiam deixar a entrevista a qualquer momento sem necessidade de explicação. $O$ estudo foi aprovado pelo Departamento de Enfermagem, Fisioterapia e Medicina, pelo Comitê de Ética em Pesquisa da Universidade de Almeria (código ENFISMED-09/16) e autorizado pela AECC.

\section{Resultados}

Dois temas principais que ajudam a entender o fenômeno das experiências sexuais após a prostatectomia radical emergiram da análise de dados (Tabela 1).

Tabela 1. Temas, subtemas e unidades de significado que emergiram da análise de dados

\begin{tabular}{|c|c|c|}
\hline Tema & Subtema & Unidades de significado \\
\hline \multirow[t]{2}{*}{$\begin{array}{l}\text { Mudanças } \\
\text { sexuais como } \\
\text { fator-chave } \\
\text { da resposta } \\
\text { adaptativa. }\end{array}$} & $\begin{array}{l}\text { Dificuldades sexuais } \\
\text { após a cirurgia }\end{array}$ & $\begin{array}{l}\text { Disfunção erétil, Desejo sexual inibido, } \\
\text { Anejaculação, Hematospermia, Ejaculação } \\
\text { retrógrada, Potência sexual, Perda de } \\
\text { sensibilidade, Bloqueio, Ginecomastia }\end{array}$ \\
\hline & $\begin{array}{l}\text { Questões com } \\
\text { relacionamentos } \\
\text { íntimos e bem-estar } \\
\text { psicossocial }\end{array}$ & $\begin{array}{l}\text { Importância da sexualidade, Resignação, } \\
\text { Espírito de melhoria, Desejo, Frustração, } \\
\text { Sexualidade como coisa do passado, } \\
\text { Masculinidade, Novas prioridades, Excitação, } \\
\text { Fuga de encontros sexuais, Interesse em } \\
\text { sexualidade }\end{array}$ \\
\hline \multirow[t]{2}{*}{$\begin{array}{l}\text { Prostatectomia: } \\
\text { a necessidade } \\
\text { de adaptação a } \\
\text { uma sexualidade } \\
\text { holística }\end{array}$} & $\begin{array}{l}\text { Questionamento das } \\
\text { experiências a partir } \\
\text { do comportamento } \\
\text { sexual atual }\end{array}$ & $\begin{array}{l}\text { Coito, Ereção, Rejeição, Satisfação, Evita pensar } \\
\text { sobre isso, Sexo, Relação entre próstata e } \\
\text { sexualidade, Diminuição da prática sexual, } \\
\text { Insatisfação sexual }\end{array}$ \\
\hline & $\begin{array}{l}\text { Adaptação da } \\
\text { sexualidade e } \\
\text { intimidade após a } \\
\text { cirurgia }\end{array}$ & $\begin{array}{l}\text { Adaptação de práticas, Após a operação, } \\
\text { Carícias, Brincadeiras, Beijos, Abraços, } \\
\text { Sexualidade no casal, Mudanças no ambiente } \\
\text { sexual }\end{array}$ \\
\hline
\end{tabular}




\section{Tema 1. Mudanças sexuais como fator-chave da resposta adaptativa}

Esse tema representa alterações na sexualidade, relacionamentos íntimos e bem-estar dos participantes após a cirurgia da próstata. Problemas na função sexual (desejo, ereção ...) se chocam com o fato de a prática sexual continuar sendo uma prioridade para os pacientes. Isso os levou a situaçóes de crise e sofrimento psicológico. Alguns participantes confirmaram a importância da sexualidade após a prostatectomia:

\section{A única coisa que me preocupa com toda a história} da próstata é isso - sexo! (P.01)

\section{Subtema 1. Dificuldades sexuais após a cirurgia}

Após uma prostatectomia radical, a diminuição da função sexual costuma ser significativa. A principal dificuldade relatada pelos participantes foi a disfunção erétil. Alguns participantes afirmaram que isso foi inesperado e que nutriam esperança de recuperaçáo com algum tipo de terapia:

Veja como estou agora. Eu costumava rir de pessoas que não conseguiam uma ereção, mas agora sou uma delas. Eu só espero superar isso e ter relaçóes normais novamente. (P.11)

Os participantes relataram mudanças que acabaram afetando o desejo sexual. Ou o desejo foi mantido, mas reprimido por problemas de ereção, ou o desejo sexual foi diretamente reduzido. De qualquer forma, os participantes evitam relaçóes sexuais com o parceiro ou mesmo discussóes sobre o assunto:

Não sinto nada, meu desejo é muito baixo, não porque eu não tenho vontade, mas porque não tenho ereçôes, então, por que vou tentar? (P.10)

Quando me perguntam sobre relaçóes sexuais com minha parceira (...), apenas evito as perguntas. No final, ela acha que eu tenho um problema com ela, e encerramos ... Não sabemos como resolver o problema. (P.13)

Um fator chave para manter um bom autoconceito é a imagem corporal. Portanto, as alteraçóes corporais secundárias às terapias adjuvantes têm um papel importante nas experiências sexuais. A deterioração das funçóes sexuais, juntamente com as mudanças na imagem corporal, gera sentimentos de vergonha em alguns participantes. Às vezes, o humor é usado como uma maneira positiva de integrar a disfunção ao cotidiano:

O fato de seu peito ficar quase igual ao de uma mulher (...) É claro que tudo isso [junto com disfunção erétil] deixa você com vergonha - já que você tem seios maiores que sua esposa, bem, não é exatamente agradável. (P.03)

\section{Subtema 2. Questões com relacionamentos íntimos e bem-estar psicossocial}

Com frequência, uma prostatectomia radical é realizada em uma idade em que os hábitos sexuais de um homem já estão profundamente enraizados. Isso significa que após a intervenção cirúrgica, os pacientes gostariam de realizar as mesmas práticas sexuais às quais estavam acostumados. Às vezes, eles podem se frustrar com as consequências físicas da prostatectomia, e isso ocorre principalmente com os pacientes que mantêm o desejo sexual intacto.

Ainda quero me divertir, mas näo consigo ter relaçôes! [...] eu quero fazer amor, mas só que não é como eu gosto (como era antes da operaçâo), eu desmorono e nem consigo me concentrar. (P.02)

O fato de não poderem retomar suas relaçóes sexuais habituais deteriora diferentes áreas da vida. Até mesmo a satisfação com a boa evolução da doença pode ser ofuscada pelo prejuízo na função sexual. Um participante viu as boas notícias manchadas de amargura.

Tudo é manchado de amargura, mesmo que você receba boas noticias. Agora, tenho que enfrentar outro desafio. Eu tenho que me preocupar porque não consigo deixar ele duro (ter ereçóes). (P.08)

Quando a atividade sexual é afetada por causas orgânicas que prejudicam o desempenho da relação sexual, a frustração dos indivíduos se torna eviden- 
te, já que a sexualidade é interpretada como algo do passado. Para eles, a sexualidade se encerra quando o coito passa a ser inviável. Alguns participantes evitam incorporar novas práticas sexuais. Assim, as consequências de uma resposta não adaptativa surgem durante a relação sexual:

Não posso ter uma ereção agora, então não consigo imaginar sexo sem isso. Então, já faz um tempo que eu nem mesmo penso em fazer sexo com minha parceira. (P.01)

Socialmente, o papel de um homem nas relaçôes heterossexuais é identificado com a relação sexual e a penetração. A prostatectomia radical não poupadora de nervos envolve uma série de mudanças na sexualidade como a conhecemos, e os participantes do estudo sentem-se incapazes de desempenhar esse papel. Isso os leva a questionamentos sobre a própria masculinidade.

Não ser capaz de ter relaçóes sexuais normais (relação sexual com penetração) muda completamente sua vida. É como ... como posso dizer? Você deixa de ser homem. (P.09)

Um participante reconheceu questionar sua própria masculinidade por não ser mais capaz de oferecer o que é tradicionalmente esperado de um homem (sustentar uma ereção). Isso gera ansiedade e conflito entre o desejo e a capacidade percebida:

[...] a ansiedade e o medo de falhar na masculinidade estão sempre rondando a situação. E luto para não evitar relaçóes sexuais porque o desejo persiste, sabe? (P.10)

\section{Tema 2. Prostatectomia: a necessidade de adaptação a uma sexualidade holística}

O tema representa as necessidades e os esforços dos participantes para adaptar suas práticas sexuais a nova situação clínica. Os participantes tentaram se adaptar à nova situação adotando novas práticas sexuais e mudando as concepçóes que vinculam a masculinidade à capacidade de ereção e penetração.

\section{Subtema 1. Questionamento das experiências a partir do comportamento sexual atual}

Alguns participantes reconhecem a necessidade de mudar a percepção tradicional, na qual a sexualidade é identificada com a relação sexual. Eles se questionam se a única forma de manter a relação sexual é através do coito e abrem suas mentes para novas práticas. No entanto, apesar do desejo, é difícil alcançar satisfação física e psicológica:

Eu sei que devo inovar e tentar novas práticas e deixar a penetração fora da minha mente. Mas para mim, ainda é difícil manter o foco em desfrutar do sexo. (P.05)

A tentativa de superar as dificuldades sexuais implica esforço para encontrar o bem-estar sexual através de uma noção mais ampla de sexualidade. Os participantes que não se adaptaram aos novos desafios notaram a mudança de sua experiência sexual.

Antes da cirurgia, eu sempre queria "fazer festa" (fazer sexo) [...] Mas agora .... Minha esposa está quase cansada disso. Quase não nos procuramos por dias, e acho que sempre superestimei essa coisa de sexo. (P.02)

\section{Subtema 2. Adaptação da sexualidade e intimidade após a cirurgia}

Os participantes relataram implementar estratégias para ajustar sua prática sexual. Além das diferentes atitudes individuais que adotaram, foi enfatizado o apoio da parceira. Segundo eles, a ajuda da parceira é indispensável para pacientes prostatectomizados, pois elas são a pessoa com quem compartilham sua intimidade.

Eu gosto de relaçóes sexuais, sem dúvida. [...] Agora, eu adaptei a prática. Isso foi muito mais fácil graças à minha parceira - agora fazemos de uma maneira diferente e nós dois podemos desfrutar. Abrir minha mente foi uma boa opção para mim. (P.07)

Carícias e abraços são maneiras de manter o contato corporal entre os casais. Essas açóes passam 


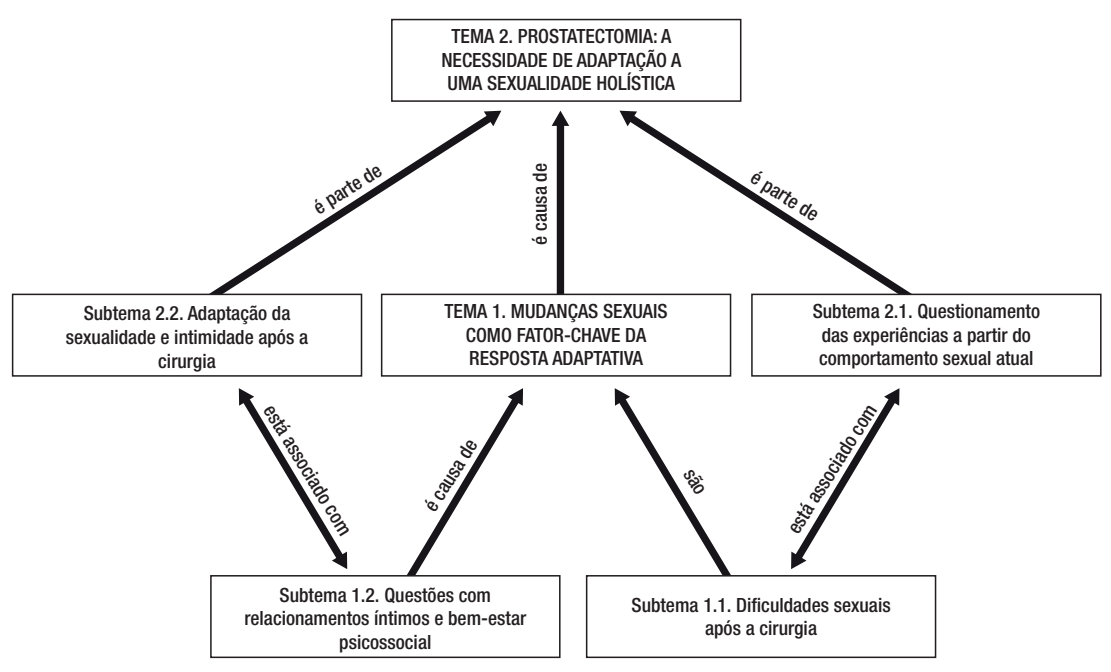

Fonte: Desenvolvido pelos autores com o ATLAS.Ti8.

Figura 1. Mapa conceitual demonstrando as relações entre temas e subtemas desenvolvidos na análise.

a desempenhar um papel importante na prática sexual até atingir uma faixa adequada de estímulo que levará a uma resposta positiva:

Antes, não havia problema com o sexo, mas agora ... Você pode acariciar, pode brincar, pode fazer outras coisas que ainda são incriveis para se sentir ativo (P.07)

Apesar da capacidade de adaptação à nova situação e de se sentirem sexualmente satisfeitos, alguns entrevistados relataram sentimento de culpa ou dúvida sobre a satisfação do parceiro em relação a sua incapacidade para o coito. $\mathrm{O}$ fato de adotar novas práticas e expandir seus hábitos sexuais não implica na mudança de concepçóes e comportamentos internalizados por anos e pode ser uma fonte de conflito no futuro:

Se não houver penetração, fazemos outra coisa, (...) ou nos esfregamos, ponto final. E nós dois aproveitamos, embora ela faça um pouco mais do que eu, porque sou eu quem não está cumprindo com tudo... (P.O1)

Em uma análise pragmática, foram estabelecidas relaçóes entre temas e subtemas e foi desenvolvido um mapa conceitual (Figure 1). Dificuldades e problemas sexuais em relacionamentos íntimos e bem-estar psicológico constituem mudanças sexuais dos pacientes submetidos a prostatectomia radical não poupadora de nervos, as quais originam ou induzem uma resposta adaptativa. Essa resposta adaptativa implica questionar os comportamentos sexuais tradicionais e buscar novas relaçôes sexuais e íntimas após a cirurgia.

\section{Discussão}

As limitações deste estudo podem surgir do diálogo sobre sexualidade e reconhecimento das próprias dificuldades sexuais de cada um, o que pode causar sentimento de vergonha e trazer informaçóes tendenciosas durante as entrevistas. Para evitar esse fato, a sexualidade foi abordada nas conversas somente após estabelecer e perceber um ambiente de confiança. Além disso, a amostra foi composta por homens heterossexuais. Essas podem ser limitaçóes ao extrapolar os resultados para homens que fazem sexo com homens, uma vez que essa população tem questões específicas não abordadas no estudo. ${ }^{(25)}$ Incluir homens com orientação sexual diferente poderia ter enriquecido os resultados.

A prostatectomia radical não poupadora de nervos tem um impacto significativo na sexualidade das pessoas. Aumentar o treinamento dos profissionais de saúde em sexologia pode se traduzir em melhores habilidades de comunicação e infor- 
mação neste campo e impactar de maneira positiva a qualidade de vida dos pacientes prostatectomizados e seus parceiros.

Vários autores destacaram as repercussóes da cirurgia da próstata em relação a diferentes aspectos da sexualidade. ${ }^{(25,26)}$ A disfunção erétil é o problema que mais preocupa os entrevistados, $\mathrm{O}$ que reforça os achados publicados por outros autores. ${ }^{(18,27)}$ A perda de libido ou inibição do desejo sexual também ocorreu em pacientes prostatectomizados, ${ }^{(28)}$ mas os participantes relacionaram a diminuição do desejo ou interesse ao aparecimento de disfunções sexuais orgânicas, como disfunção erétil. Ecoando achados de outros autores, ${ }^{(29)}$ nossos participantes relataram o aparecimento de disfunçóes ejaculatórias. Embora todas essas disfunções sexuais tenham sido amplamente discutidas na literatura, ${ }^{(28-31)}$ nosso estudo enfatiza seu impacto nas relaçóes pessoais e no bem-estar psicossocial. Questôes relacionadas à saúde sexual e reprodutiva têm um papel importante na formação da identidade pessoal e de gênero. ${ }^{(32,33)}$ Este estudo sugere que a disfunção sexual masculina de pacientes submetidos a prostatectomia radical não poupadora de nervos também pode prejudicar sua masculinidade e identidade como homens.

Alguns pacientes acreditam que a atividade sexual é coisa do passado, enquanto outros buscam a adaptação no sentido de restabelecer e recuperar suas funçóes sexuais. Nesse sentido, alguns autores sugeriram uma adaptaçáo dos objetivos relacionados à qualidade de vida de acordo com a idade e as prioridades individuais. ${ }^{(30)}$ A adoção de técnicas poupadoras de nervos para prostatectomia radical, juntamente com terapia farmacológica adjuvante, tem alcançado resultados esperançosos na reabilitação sexual. ${ }^{(26)}$ No entanto, ainda não há evidências claras para recomendar um algoritmo irrefutável para reabilitação peniana após prostatectomia. ${ }^{(27)}$ Além disso, contrariamente às expectativas da maioria dos entrevistados, a restauração da função sexual nem sempre foi relacionada à satisfação sexuaI. ${ }^{(31)}$ Este estudo sugere a importância do aconselhamento pré-prostatectomia para ajudar os pacientes a gerenciar suas expectativas. Além disso, intervenções destinadas à adaptação e práticas sexuais não focadas na ereção e penetração podem ser úteis.

Os participantes mostraram frustração ou resignação ao buscar a adaptação a uma nova prática sexual, enquanto alguns autores relataram a possibilidade de um nível de satisfação aceitável e um nível de satisfação ainda melhor do que antes da cirurgia. ${ }^{(29)}$ Outros autores destacaram a importância dos parceiros na superação de problemas sexuais após a prostatectomia ${ }^{(20)}$, o que consequentemente, melhora o nível de adaptação dos pacientes. ${ }^{(33)}$

Os avanços importantes trazidos para a enfermagem e outros profissionais da saúde nesse estudo têm relação com a atenção dedicada ao bem-estar sexual dos pacientes submetidos a prostatectomia. As disfunçóes sexuais derivadas da prostatectomia radical não poupadora de nervos podem afetar as relaçóes interpessoais e o bem-estar psicossocial. Uma formação que englobe aspectos da resposta sexual humana pode ajudar os profissionais a oferecer uma assistência abrangente aos pacientes com doenças na próstata. Embora o cuidado interdisciplinar seja mencionado em outros estudos, ele se limita à psicologia e à medicina. ${ }^{(4,31)} \mathrm{O}$ presente estudo sugere que, assim como em outras síndromes que afetam a sexualidade, ${ }^{(32)}$ os enfermeiros podem ajudar na adaptação bem-sucedida aos problemas sexuais relacionados com a prostatectomia radical não poupadora de nervos.

\section{Conclusão}

Os pacientes submetidos a prostatectomia radical não poupadora de nervos enfrentam dificuldades sexuais, como disfunção erétil ou desejo sexual hipoativo. Essas mudanças causam problemas nos relacionamentos sexuais e íntimos, que afetam o bem-estar psicossocial e levam a sentimentos de resignação ou frustração. Como essa situação afeta os pacientes e o casal, alguns participantes se questionam sobre suas práticas sexuais habituais. Eles sentem a necessidade de adaptação a uma sexualidade holística, não centrada no coito e que incorpore formas inovadoras de sexualidade para o casal. 


\section{Agradecimentos}

Agradecemos a todos os que participaram deste estudo. Esta pesquisa foi financiada pelo Grupo de Pesquisa em Ciências da Saúde (CTS-451) e pelo Centro de Investigação em Saúde (CEINSA) da Universidade de Almeria, Espanha.

\section{Colaborações}

Fernández-Sola C, Martínez-Bordajandi A e Hernández-PadillaJM foram responsáveis pelodesenho do estudo. Puga-Mendoza AP, López-Entrambasaguas OM, Lucas-Matheu M realizaram a coleta de dados. Granero-Molina J, López-Entrambasaguas OM, e Fernández-Medina IM planejaram e realizaram a análise e interpretação dos dados. Fernández-Sola C, Granero-Molina J, Hernández-Padilla JM e MartínezBordajandi A redigiram o manuscrito, e Lucas-Matheu OM, Puga-Mendoza AP, Fernández-Medina IM e López-Entrambasaguas OM fizeram a revisão crítica. Todos os autores examinaram o manuscrito quanto à precisão e integridade.

\section{Referências}

1. llic D, Djulbegovic M, Jung JH, Hwang EC, Zhou Q, Cleves A, et al. Prostate cancer screening with prostate-specific antigen (PSA) test: a systematic review and meta-analysis. BMJ. 2018 ;362:k3519.

2. Culp MB, Soerjomataram I, Efstathiou JA, Bray F, Jemal A. Recent global patterns in prostate cancer incidence and mortality rates. Eur Urol. 2020;77(1):38-52.

3. Etxeberria J, Guevara M, Moreno-Iribas C, Burgui R, Delfrade I, Floristán $Y$, et al. Prostate cancer incidence and mortality in Navarre (Spain). An Sist Sanit Navar. 2018;41(1):9-15.

4. Bolla M, Henry A, Mason M, WiegelT. The role of radiotherapy in localised and locally advanced prostate cancer. Asian J Urol. 2019;6(2):153-61.

5. Hatiboglu G, Popeneciu V, Bonekamp D, Burtnyk M, Staruch R, Pahernik $S$, et al. Magnetic resonance imaging-guided transurethral ultrasound ablation of prostate tissue in patients with localized prostate cancer: singlecenter evaluation of 6-month treatment safety and functional outcomes of intensified treatment parameters. World J Urol. 2020;38(2):343-50.

6. Gravis G. Systemic treatment for metastatic prostate cancer. Asian J Urol. 2019;6(2):162-8.

7. Bill-Axelson A, Holmberg L, Garmo H, Taari K, Busch C, Nordling S, Häggman M, Andersson SO, Andrén 0 , Steineck G, Adami HO, Johansson JE. Radical prostatectomy or watchful waiting in prostate cancer - 29-year follow-up. N Engl J Med. 2018;379(24):2319-29.
8. Morlacco A, Karnes RJ. High-risk prostate cancer: the role of surgical management. Crit Rev Oncol Hematol. 2016;102:135-43.

9. Galfano A, Secco S, Panarello D, Di Trapani D, Strada E, Petralia G, et al. Radical prostatectomy through the posterior technique. In: John $\mathrm{H}$, Wiklund P, editors. Robotic urology. Cham: Springer International Publishing; 2018. p. 401-10.

10. Shpot EV, Chinenov DV, Amosov AV, Chernov YN, Yurova MV, Lerner YV. [Erectile dysfunction associated with radical prostatectomy: appropriateness and methods to preserve potency]. Urologiia. 2018;2_2018(2):75-82. Russian.

11. Chambers SK, Chung E, Wittert G, Hyde MK. Erectile dysfunction, masculinity, and psychosocial outcomes: a review of the experiences of men after prostate cancer treatment. Transl Androl Urol. 2017;6(1):60-8.

12. Fode M, Serefoglu EC, Albersen M, Sønksen J. Sexuality following radical prostatectomy: is restoration of erectile function enough? Sex Med Rev. 2017;5(1):110-9.

13. Lehto US, Tenhola H, Taari K, Aromaa A. Patients' perceptions of the negative effects following different prostate cancer treatments and the impact on psychological well-being: a nationwide survey. $\mathrm{Br} \mathrm{J}$ Cancer. 2017;116(7):864-73.

14. Grondhuis Palacios LA, Krouwel EM, Duijn M, den Oudsten BL, den Ouden ME, Putter $\mathrm{H}$, et al. Written information material and availability of sexual health care for men experiencing sexual dysfunction after prostate cancer treatment: an evaluation of Dutch urology and radiotherapy departments. Eur $\mathrm{J}$ Cancer Care (Engl). 2017;26(2):e12629.

15. Okçelik S, Ates F, Yazıcı V, Soydan H, Balıbey H, Karademir K. Erectile dysfunction after radical prostatectomy: effect of quality of life and psychology. Rev Int Androl. 2015;13(4):120-4.

16. Katz A, Dizon DS. Sexuality after cancer: a model for male survivors. J Sex Med. 2016;13(1):70-8.

17. Wittmann D, Carolan M, Given B, Skolarus TA, Crossley H, An L, et al. What couples say about their recovery of sexual intimacy after prostatectomy: toward the development of a conceptual model of couples' sexual recovery after surgery for prostate cancer. J Sex Med. 2015;12(2):494-504.

18. Blomberg $K$, Wengström $Y$, Sundberg $K$, Browall $M$, Isaksson AK, Nyman $\mathrm{MH}$, et al. Symptoms and self-care strategies during and six months after radiotherapy for prostate cancer - Scoping the perspectives of patients, professionals and literature. Eur J Oncol Nurs. 2016;21:139-45.

19. Ernstmann N, Weissbach L, Herden J, Winter N, Ansmann L. Patientphysician communication and health-related quality of life of patients with localised prostate cancer undergoing radical prostatectomy - a longitudinal multilevel analysis. BJU Int. 201;119(3):396-405.

20. Parahoo K, McKenna S, Prue G, McSorley 0, McCaughan E. Facilitators' delivery of a psychosocial intervention in a controlled trial for men with prostate cancer and their partners: a process evaluation. J Adv Nurs. 2017;73(7):1620-31.

21. McCaughan E, McKenna S, McSorley 0, Parahoo K. The experience and perceptions of men with prostate cancer and their partners of the CONNECT psychosocial intervention: a qualitative exploration. J Adv Nurs. 2015;71(8):1871-82.

22. Fleming V, Robb Y. A critical analysis of articles using a Gadamerian based research method. Nurs Inq. 2019;26(2):e12283.

23. Fernández-Sola C, Huancara-Kana D, Granero-Molina J, CarmonaSamper E, López-Rodríguez MM, Hernández-Padilla JM. Sexualidade durante todas as fases da gravidez: experiências de gestantes. Acta Paul Enferm. 2018;31(3):305-12. 
24. Buus N, Perron A. The quality of quality criteria: Replicating the development of the Consolidated Criteria for Reporting Qualitative Research (COREQ). Int J Nurs Stud. 2019;102:103452.

25. Lee TK, Handy AB, Kwan W, Oliffe JL, Brotto LA, Wassersug RJ, et al. Impact of prostate cancer treatment on the sexual quality of life for men-who-have-sex-with-men. J Sex Med. 2015;12(12):237886.

26. Siena G, Mari A, Canale A, Mondaini N, Chindemi A, Greco I, et al. Sexual rehabilitation after nerve-sparing radical prostatectomy: free-of-charge phosphodiesterase type 5 inhibitor administration improves compliance to treatment. J Sex Med. 2018;15(2):1203.

27. Clavell-Hernandez J, Wang R. Penile rehabilitation following prostate cancer treatment: review of current literature. Asian $\mathrm{J}$ Androl. 2015;17(6):916-22.

28. Haney NM, Alzweri LM, Hellstrom WJ. Male orgasmic dysfunction postradical pelvic surgery. Sex Med Rev. 2018;6(3):429-37.
29. Bang SL, Almallah YZ. The impact of post-radical prostatectomy urinary incontinence on sexual and orgasmic well-being of patients. Urology. 2016;89:1-5

30. Hampson LA, Cowan JE, Zhao S, Carroll PR, Cooperberg MR. Impact of age on quality-of-life outcomes after treatment for localized prostate cancer. Eur Urol. 2015;68(3):480-6.

31. Terrier JE, Masterson M, Mulhall JP, Nelson CJ. Decrease in intercourse satisfaction in men who recover erections after radical prostatectomy. J Sex Med. 2018;15(8):1133-9.

32. Matarín Jiménez TM, Fernández-Sola C, Hernández-Padilla JM, Correa Casado M, Antequera Raynal LH, Granero-Molina J. Perceptions about the sexuality of women with fibromyalgia syndrome: a phenomenological study. J Adv Nurs. 2017;73(7):1646-56.

33. Fernández-Sola C, Martínez-Caba Ml, Hernández-Padilla JM, Carmona-Samper E, Granero-Molina J. Experiences of Spanish women undergoing hysterosalpingography as part of the infertility process: a phenomenological study. J Clin Nurs. 2016;25(3-4):494-504. 